\title{
Towards a high-resolution TOF- MIEZE spectrometer with very cold neutrons
}

\section{$\operatorname{AUTHOR}(\mathrm{S})$ :}

Oda, T.; Hino, M.; Kitaguchi, M.; Filter, H.; Geltenbort, P.; Kawabata, Y.

\section{CITATION:}

Oda, T.... [et al]. Towards a high-resolution TOF-MIEZE spectrometer with very cold neutrons. Nuclear Instruments and Methods in Physics Research Section A: Accelerators, Spectrometers, Detectors and Associated Equipment 2017, 860: 35-41

ISSUE DATE:

2017-07-11

URL:

http://hdl.handle.net/2433/259242

\section{RIGHT:}

(c) 2017. This manuscript version is made available under the CC-BY-NC-ND 4.0 license

http://creativecommons.org/licenses/by-nc-nd/4.0/; The full-text file will be made open to the public on 1 December 2020 in accordance with publisher's 'Terms and Conditions for Self-Archiving'; この論文は出版社版でありません。引用 の際には出版社版をご確認じ利用ください。; This is not the published version. Please cite only the published version. 
Accepted by Nucl. Instrum. Methods A DOI: 10.1016/j.nima.2017.03.014

\title{
Towards a high-resolution TOF-MIEZE spectrometer with very cold neutrons
}

\author{
T. Oda ${ }^{\mathrm{a}, 1}$, M. Hino ${ }^{\mathrm{b}}$, M. Kitaguchi ${ }^{\mathrm{c}, \mathrm{d}}$, H. Filter ${ }^{\mathrm{e}}$, P. Geltenbort ${ }^{\mathrm{f}}$, \\ Y. Kawabatab \\ ${ }^{a}$ Department of Nuclear Engineering, Kyoto University, Kyoto 615-8540, Japan \\ ${ }^{b}$ Research Reactor Institute, Kyoto University, Kumatori 590-0494, Japan \\ ${ }^{c}$ Department of Physics, Nagoya University, Chikusa, Nagoya 464-8602, Japan \\ ${ }^{d}$ Center for Experimental Studies, KMI, Nagoya University, Nagoya 464-8602, Japan \\ ${ }^{e}$ Atominstitut, Technische Universität Wien, 1020 Wien, Austria \\ ${ }^{f}$ Institut Laue Langevin, 38042 Grenoble, France
}

\begin{abstract}
We report the first experimental test of TOF-MIEZE technique using very cold neutrons (VCNs) towards high-resolution quasi-elastic neutron scattering spectroscopy. TOF-MIEZE is a type of neutron resonance spin echo spectroscopy with a combination of the time-of-flight (TOF) method and modulation of intensity by zero effort (MIEZE). A compact MIEZE instrument was constructed at the VCN beam port at the High Flux Reactor at the Institut Laue Langevin. By accumulating individual oscillations of raw data, we observed a TOF-MIEZE signal with an effective frequency of 50 $\mathrm{kHz}$ in a wavelength band of $4-6 \mathrm{~nm}$. The signal contrasts were $0.59 \pm 0.04$ and $0.29 \pm 0.03$ for wavelengths of $4 \mathrm{~nm}$ and $6 \mathrm{~nm}$, respectively. The Fourier time was estimated to be $70 \mathrm{~ns}$ with $6 \mathrm{~nm}$ VCNs in the experimental set-up. Keywords: Neutron spin echo, MIEZE, time-of-flight method, very cold neutron, pulsed neutron beam

\footnotetext{
Email address: t_oda@rri.kyoto-u.ac.jp (T. Oda)

${ }^{1}$ Present address: Research Reactor Institute, Kyoto University, Kumatori 590-0494, Japan
} 


\section{Introduction}

Slow neutrons in the energy range from approximately $2 \times 10^{-7} \mathrm{eV}$ to $5 \times 10^{-5} \mathrm{eV}$ are classified as very cold neutron (VCN) [1]. VCN has played an important role in neutron optics, e.g., spin interferometers [2] and holographic grating interferometers [3], and in fundamental physics research [4], e.g., measuring gravitational phase shifts [5]. Even in large-scale neutron facilities, however, VCN beams have seldom been used in spectrometers for material science due to the low intensity of VCNs from typical Maxwellian distributions of neutron source spectra and the relatively high absorption of VCN in most materials.

Expanding the wavelength range of use into VCN will provide benefits in particular techniques of neutron scattering for material science. The reflection and refraction angles of neutrons increase with increasing wavelength. Therefore, VCNs have large reflection angles and the recent progress of reflective neutron devices [6-12] will enable us to handle VCN beams in a manner similar to optical beams and compensate the low intensity. Using a VCN beam, it is possible to investigate sample structures at a mesoscopic scale efficiently with sample focusing conditions; this avoids any unnecessary Bragg scattering from crystalline structures of the sample since the VCN wavelength is longer than the Bragg cut-off wavelength of the sample. The combination of a focused VCN beam and small angle neutron scattering (SANS) has the advantage of reaching lower momentum transfers, $Q$, which corresponds to the ability to observe larger structural scales. Using this advantage, a technique involving polarized VCN-SANS and a time-of-flight (TOF) method has been successfully tested with an advanced magnetic lens system [13].

The neutron is a useful probe to study the dynamics of condensed matters as well as the static structures. Neutron spin echo (NSE) spectroscopy [14-16] is a unique method for quasi-elastic neutron scattering spectroscopy and has the highest energy resolution of existing neutron spectrometers. In conventional NSE instruments, two strong static magnetic fields are placed before and after the sample position. Reversal Larmor precession of the neutron spin are realized by these magnetic fields and a $\pi$-flipper. Through the spin manipulations, the neutron is in a superposition of the spin-up and down eigenstates, and the phase difference between the two states corresponds the Larmor precession angle. Labeling the velocity of the neutron with the precession angle makes it possible for us to observe minute changes in neutron velocity after interaction with a sample. In NSE spectroscopy, the interme- 
diate scattering function, $S(\boldsymbol{Q}, \tau)$, is directly obtained and the energy resolution is decoupled from the wavelength distribution of the incident beam.

The modulation of intensity by zero effort (MIEZE) technique is a remarkable variation of the NSE method [17]. Neutron spin manipulation for MIEZE instruments are made by resonance spin flippers (RSFs), which consists of a radio-frequency (RF) oscillating magnetic field and static field. Neutrons exchange an energy corresponding to the RF via a transition from one spin eigenstate to another (spin flip) by RSFs. Coherent states with an energy difference is realized by a set of RSFs operated in different frequencies. After a spin analyzer, we can observe a time-dependent neutron intensity modulation with a frequency corresponding the energy difference. In contrast to the conventional spin ehco method, simple MIEZE spectrometers need no polarization components after the sample. The essential spin analyzer is placed before the sample and the neutron in the spin-up eigenstate (with an energy difference) enters the sample; this feature allows great flexibility of the sample environment, such as applying a high magnetic field at the sample [18, 19], and also allows polarimetry for the magnetic [20] or incoherent scattering [21, 22].

NSE instruments measure a contrast decreasing of the spin echo signal caused by inelastic scattering, and the index of their energy resolution is called Fourier time. The Fourier time of a typical MIEZE set-up, $\tau_{\mathrm{M}}$, is given by

$$
\tau_{\mathrm{M}}=\frac{\hbar L_{\mathrm{sd}}}{m_{\mathrm{n}} v^{3}} \omega_{\mathrm{M}}
$$

where $m_{\mathrm{n}}$ is the neutron mass, $v$ is neutron velocity, $L_{\mathrm{sd}}$ is the flight path length between the sample and detector, and $\hbar \omega_{\mathrm{M}}$ is the effective energy difference caused by the resonance spin flippers (RSFs). The Fourier time is proportional to $L_{\mathrm{sd}}, \omega_{\mathrm{M}}$ and $v^{-3}$. The VCN beam has the advantage of a higher resolution measurement since the energy resolution of the MIEZE method is proportional to the third power of neutron wavelength. In the geometry of the MIEZE measurements, there is a severe limitation of the dispersion of path lengths of the neutron beams. The contrast of MIEZE signals can be drastically reduced by the path length dispersion depending on the sample shapes [23 25] and the effective thickness of the detector, particularly with high frequencies. In SANS or neutron reflectometry (NR) geometries, this geometrical limitation is alleviated [25, 26]. Several experiments on the quasi-elastic scattering using MIEZE type neutron spin echo spectrom- 
eter have been performed in steady state neutron sources [18, 19, 23, 27]. Resonance-type neutron spin echo spectrometers with MIEZE option, named RESEDA [28] and MIRA [29] are currently operated at MLZ, Garching.

By including the TOF information with the MIEZE technique (TOFMIEZE), it is possible to cover a wide range of momentum transfers and energy exchanges simultaneously. Furthermore, the contrast of the TOFMIEZE signal is robust against experimental misalignments with a short pulsed beam [30. The simple MIEZE configuration will allow a MIEZEoption on existing SANS or NR instruments to investigate not only static structures, but also dynamics that relates to functional properties of the sample. Several efforts have been made to establish the TOF-MIEZE technique for innovative studies of the slow dynamics of condensed matter systems [31, 32].

In the TOF-MIEZE technique, differently from the other NSE methods, the echo signal is plotted as a function of time, and neutron wavelength is also distinguished by time. Typically, the time scale of the period of the MIEZE signal $(\sim 1 \mu \mathrm{s})$ is much smaller than the whole TOF range $(\sim$ $1 \mathrm{~ms})$. The low neutron count statistics accompanies the fine binning of the time channel for the MIEZE signals. A straightforward method to obtain a TOF-MIEZE signal with good statistics is to accumulate neutron counts by summing multiple periods of the MIEZE signal. In Ref. [32] a summation of 12 oscillations of the TOF-MIEZE signal was used to improve the statistics in calculating signal contrast.

In this study, we show the first TOF-MIEZE signal with a pulsed VCN beam whose wavelength is longer than $4 \mathrm{~nm}$ and demonstrate the validity of simple data accumulation in TOF-MIEZE measurements. By accumulating over a time range of 100 times larger than the MIEZE periods, a TOFMIEZE signal was successfully reproduced from raw data that have no visible signals since each time channel of the raw data has insufficient neutron count statistics.

\section{Measurement of the primary VCN beam profile}

PF2/VCN at the High Flux Reactor (HFR) at Institut Laue Langevin (ILL) is a unique facility that provides continuous beam of VCNs. The experimental MIEZE instrument was installed in the Level-D section of the reactor hall. The $\mathrm{VCN}$ beam is transported through the vertical guide tube from a cold source [33]. 


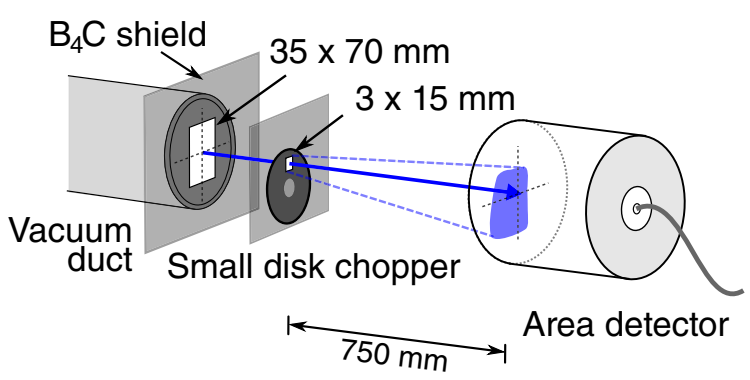

Figure 1: Measurement geometry of the primary VCN beam profile in the upstream part of the PF2/VCN.

Before performing the MIEZE-type spin echo experiments, we measured the primary VCN beam profile in order to estimate the count rate of the VCNs. Figure 1 shows the schematic layout of the primary VCN beam measurement in the upstream part of the beam port. The beam divergence was determined by a $3 \times 15 \mathrm{~mm}^{2}$ aperture placed just after a small disk chopper. The distance between the small chopper and detector was $750 \mathrm{~mm}$. The center of the detector was placed in line with the vacuum duct exit. The spatial and wavelength distribution of the VCN beam are shown in Figure 2. We separated the detector plane into four regions, A1, A2, B1, and B2 indicated by the grid in Fig. 2a. Figure 2b, shows the wavelength spectra of each of the four regions and the total wavelength spectrum. The most intense region of the beam was the B1 region of the Fig. 2a. The wavelength of the peak intensity was approximately $3 \mathrm{~nm}$ and the beam direction was downwards to the experimental room floor. The direction of the VCN beam with a longer wavelength of approximately $6 \mathrm{~nm}$ were parallel to the floor. In the TOFMIEZE experiment, we produced a beam parallel to the experimental room floor to create a VCN beam with longer wavelengths, around $6 \mathrm{~nm}$.

\section{Experimental set-up and devices}

Figure 3 shows the layout of the TOF-MIEZE experiment. The total flight path length was $L_{0 \mathrm{~d}}=3.0 \mathrm{~m}$. The distance between the chopper and RSF1 was $L_{01}=1.0 \mathrm{~m}$, the RSF1-RSF2 distance was $L_{12}=1.5 \mathrm{~m}$, and the RSF2-detector distance was $L_{2 \mathrm{~d}}=1.0 \mathrm{~m}$. A helium-filled flight tube was placed between the two RSFs to reduce VCN intensity attenuation by air. A disk chopper was installed to simulate a pulsed neutron source for TOFMIEZE technique. The rotating frequency of the chopper was set to $12.5 \mathrm{~Hz}$ 


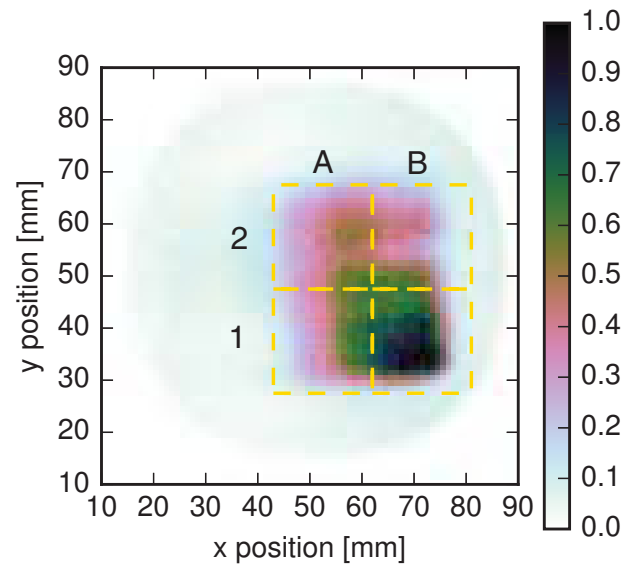

(a) Two-dimensional relative intensity map of the primary VCN beam integrated over the whole wavelength range, up to $12 \mathrm{~nm}$. We divided the illuminated area into left, right, bottom, and top sides denoted by A, B, 1, and 2 , respectively.

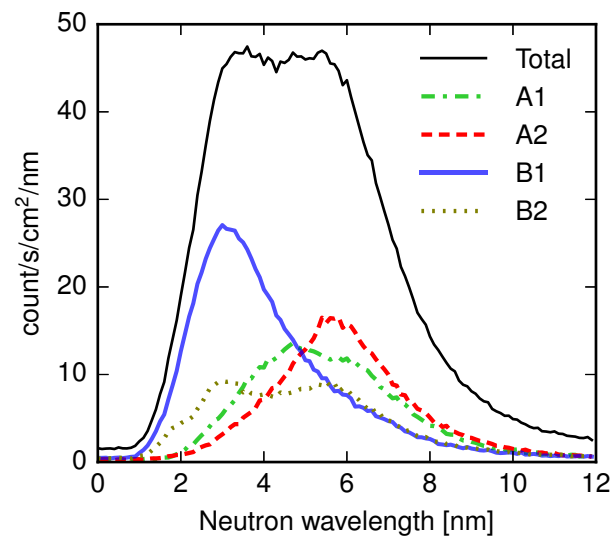

(b) Wavelength spectra in each region of the detector plane as labeled in (a)

Figure 2: Referential measurement data of the primary VCN beam for the TOF-MIEZE experiment. 


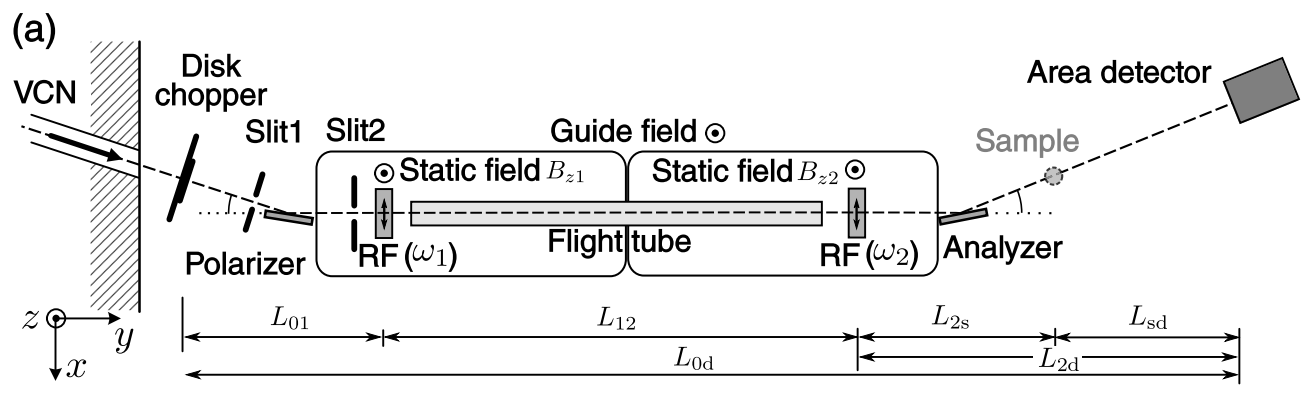

(b)

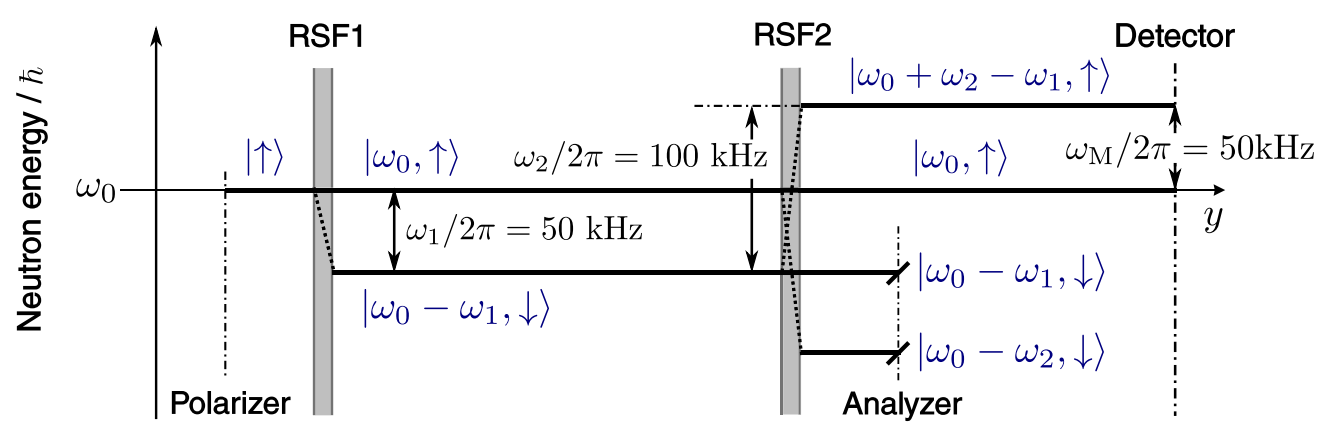

Figure 3: (a) Schematic of the top view of the experimental TOF-MIEZE set-up. $\omega_{1}$ and $\omega_{2}$ are the radio-frequencies of the first and second resonance spin flippers (RSF1 and RSF2). $L_{01}, L_{12}, L_{2 \mathrm{~s}}, L_{\mathrm{sd}}$, and $L_{2 \mathrm{~d}}$ denote the chopper-RSF1, RSF1-RSF2, RSF2sample position, sample position-detector, and RSF2-detector distances, respectively. $L_{0 \mathrm{~d}}$ denotes the total flight path length from the chopper to the detector. The orientation of the static fields and the guide field were set to the $z$ direction. The RF oscillated in the $x$ direction. (b) Energy diagram of the neutron along with the beam direction. The $|\uparrow\rangle$ and $|\downarrow\rangle$ are notations for the spin-up and -down eigenstate, respectively. $\hbar \omega_{0}$ denotes the kinetic energy of the incident neutron in free space. The quantum states of the neutron are also labeled by possible energy values. 
to cover a TOF frame up to $80 \mathrm{~ms}$. With the flight path $L_{0 \mathrm{~d}}=3.0 \mathrm{~m}$, the measurable maximum wavelength was approximately $10 \mathrm{~nm}$. The chopper disk was coated with gadolinium oxide $\left(\mathrm{Gd}_{2} \mathrm{O}_{3}\right)$ as a neutron absorber. The pulse time width was $2.7 \mathrm{~ms}$. To select one neutron spin eigenstate before and after the spin manipulations using RSFs, we used $m=4$, Fe/SiGe super-mirrors as the polarizer and analyzer. The $m$-value of a super-mirror denotes that its critical momentum transfer upon reflection is $m$ times higher than a Ni mirror only for neutrons in the spin-up state. The super-mirror multilayer was coated on a silicon wafer using ion beam sputtering technique in the Research Reactor Institute, Kyoto University [34]. We used a circular, time-sensitive area detector. The detector consisted of a 5" position-sensitive photomultiplier and a ${ }^{6} \mathrm{Li}$-enriched glass scintillator with a thickness of $0.2 \mathrm{~mm}$ [35].

\subsection{Resonance spin flippers for a pulsed neutron beam}

We used two RSFs, each consisting of orthogonal static and oscillating magnetic fields. The transition between neutron spin states occurs when the magnitude of the static magnetic field, $B_{z}$, satisfies the resonance condition:

$$
2\left|\mu_{\mathrm{n}}\right| B_{z}=\hbar \omega
$$

where $\left|\mu_{\mathrm{n}}\right|$ is the absolute value of the neutron magnetic moment, $\hbar$ is the reduced Planck's constant and $\omega$ is the angular frequency of the oscillating field. The time evolution of such a two-level spin system in a RF filed is known as Rabi oscillation, and the probability of a spin-flip is given by [36]

$$
\begin{aligned}
& P=A \sin ^{2}\left(\frac{\Theta_{\mathrm{r}}(v)}{\sqrt{A}}\right), \\
& A=\frac{\left(\left|\mu_{\mathrm{n}}\right| B_{\mathrm{r}}\right)^{2}}{\left(\left|\mu_{\mathrm{n}}\right| B_{\mathrm{r}}\right)^{2}+\hbar^{2}\left(\omega_{z}-\omega / 2\right)^{2}}, \Theta_{\mathrm{r}}(v)=\frac{\left|\mu_{\mathrm{n}}\right| B_{\mathrm{r}}}{\hbar} \frac{\ell}{v}
\end{aligned}
$$

where $B_{\mathrm{r}}$ is the amplitude of oscillating field, $\ell$ is the length of the RF interaction region. The angular frequency, $\omega_{z}$ is defined as $\left|\mu_{\mathrm{n}}\right| B_{z} / \hbar$. When $P=1$ and $P=1 / 2$, they are called $\pi$-flip and $\pi / 2$-flip, respectively. With the $\pi / 2$-flip condition, an incident neutron forms a coherent superposition state of spin-up and spin-down with a certain phase.

In a resonance spin flip process, a neutron loses energy $\hbar \omega$, for the spin-up to spin-down flip, while a neutron gains energy $\hbar \omega$, for the spin-down to spinup flip. On resonance, $A$ in Eq. (4) is unity and we have $P=\sin ^{2}\left(\Theta_{\mathrm{r}}(v)\right)$. By changing the amplitude of the oscillating field to satisfy the following 


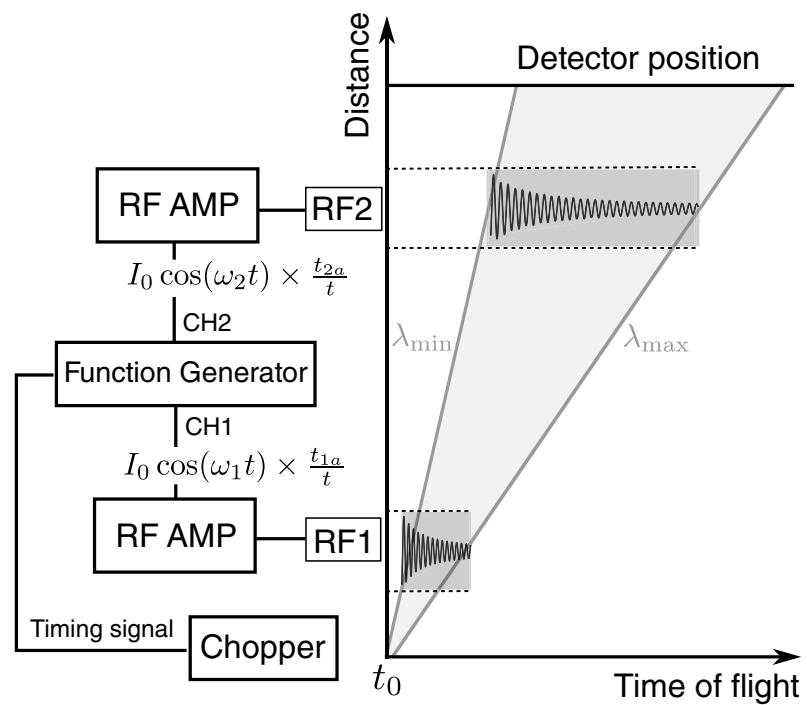

Figure 4: Time-of-flight diagram with illustrations of the time-modulated RF current and schematic layout of the electronic equipment.

equation, it is possible to use the RSF as a $\pi / 2$ flipper for a pulsed white beam [37, 38].

$$
\Theta_{\mathrm{r}}(v)=\frac{\pi}{4} \Longleftrightarrow \frac{\left|\mu_{\mathrm{n}}\right| B_{\mathrm{r}}}{\hbar} \frac{t}{t_{i a}}=\frac{\pi}{4},
$$

where $t_{i a}$ is the arrival time at each $\operatorname{RSF}(i=1,2)$. Figure 4 shows a schematic diagram of the RF amplifier system. A function generator with two channels was used to produce the time-modulated sinusoidal function $\left(\cos \left(\omega_{i} t\right) \times 1 / t\right)$. The RSF1 and RSF2 shared the same trigger signal to lock their relative phase of the RF field.

\subsection{MIEZE condition}

In the simple MIEZE instrument, both RSFs were operated as $\pi / 2$ flippers and we obtained coherent states in the spin-up eigenstate with an energy difference of $\hbar\left(\omega_{2}-\omega_{1}\right)$ at the detector as shown Figure 3 (b). The MIEZE signal is observed as a function of the phase difference between two energy states [39]. The neutron intensity at the detector position is modulated by

$$
I \propto \frac{1+\cos \phi}{2},
$$


where $\phi$ is the phase difference between the coherent spin-up states with different energies.

With no change in the neutron velocity, the phase difference at a time point $t_{\mathrm{d}}$ is given by

$$
\phi\left(t_{\mathrm{d}}\right)=-\left(\omega_{2}-\omega_{1}\right) t_{\mathrm{d}}-\frac{\omega_{1}}{v} L_{12}+\frac{\omega_{2}-\omega_{1}}{v} L_{2 \mathrm{~d}},
$$

where $\omega_{1}$ and $\omega_{2}$ are the frequencies of RSF1 and RSF2, respectively. In order to cancel out dispersive velocity-dependent phase differences, the following condition is required

$$
\omega_{1} L_{12}=\left(\omega_{2}-\omega_{1}\right) L_{2 \mathrm{~d}}
$$

This is called the MIEZE condition. In this experiment, the frequencies of RSF1 and RSF2 were set to $\omega_{1} / 2 \pi=50 \mathrm{kHz}$ and $\omega_{2} / 2 \pi=100 \mathrm{kHz}$, and the alignment constraint was $L_{12}=L_{2 \mathrm{~d}}$. In the MIEZE condition, the phase difference only depends on the detection time, $t_{\mathrm{d}}$ and we detect a neutron intensity modulation as a sinusoidal function of $t_{\mathrm{d}}$, with a frequency of $\omega_{\mathrm{M}}=$ $\omega_{2}-\omega_{1}$ :

$$
I\left(t_{\mathrm{d}}\right)=\int \mathrm{d} v \frac{f_{0}(v)}{2}\left[1+\cos \left(\omega_{\mathrm{M}} t_{\mathrm{d}}\right)\right]
$$

where $f_{0}(v)$ denotes the spectrum of the direct beam.

\section{Results and discussion}

We tested the MIEZE condition with a monochromatic neutron beam whose wavelength was $\lambda=5.0 \mathrm{~nm}$. The VCN beam was monochromated by a multilayer monochromator and the wavelength resolution was $\Delta \lambda / \lambda \approx 6 \%$.

The lengths $L_{12}$ and $L_{2 \mathrm{~d}}$ were set to $1.5 \mathrm{~m}$ and $1.0 \mathrm{~m}$, respectively, to obtain the highest contrast. Note that the discrepancy between the theoretical relation $\left(L_{12}=L_{2 \mathrm{~d}}\right)$ and the actual set-up of the MIEZE condition is due to the existence of the guide field between RSF1 and RSF2. The magnitude of the guide field was approximately $0.6 \mathrm{mT}$.

Figure 5 shows a MIEZE signal with an effective frequency of $50 \mathrm{kHz}$ with the monochromatic beam. The position of the detector was scanned with a $1 \mathrm{~mm}$ step. The solid lines are sinusoidal fitted curves: $y(x)=$ $B+A \sin \left(2 \pi x / T+\phi_{0}\right)$. The fitting parameters and calculated contrasts 


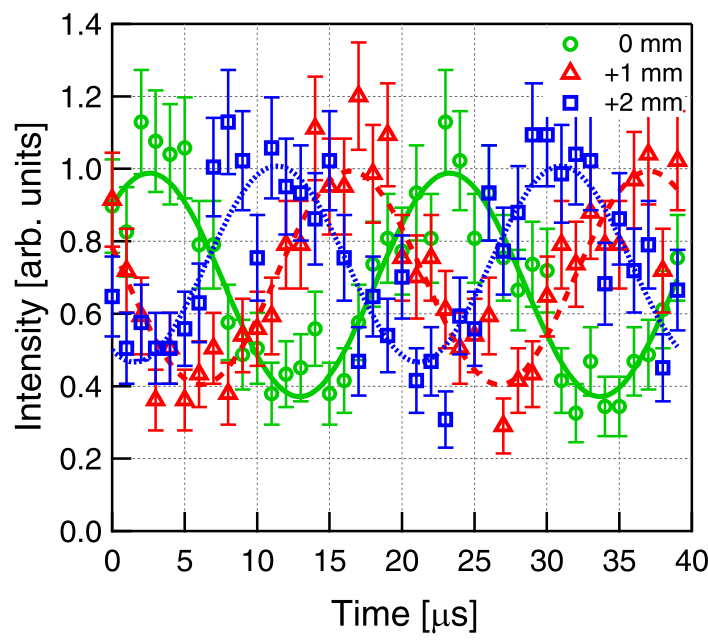

Figure 5: Phase shift of a MIEZE signal with a monochromatic beam $(\lambda=5 \mathrm{~nm})$ by scanning the detector position shown by detecting the intensity as a function of time. The solid lines are sinusoidal fitted curves. The circle, square, and triangle symbols correspond to detector positions increasing by $1.0 \mathrm{~mm}$ increments.

Table 1: Fitting parameters and calculated contrasts at different detector positions. The fit function was $y(x)=B+A \sin \left(2 \pi x / T+\phi_{0}\right)$.

\begin{tabular}{cccc}
\hline \hline Displacement & Contrast, $A / B$ & $T[\mu \mathrm{s}]$ & $\phi_{0}[\mathrm{rad}]$ \\
\hline $0 \mathrm{~mm}$ & $0.45 \pm 0.04$ & $20.7 \pm 0.5$ & $0.79 \pm 0.17$ \\
$1 \mathrm{~mm}$ & $0.42 \pm 0.04$ & $20.6 \pm 0.5$ & $2.85 \pm 0.16$ \\
$2 \mathrm{~mm}$ & $0.37 \pm 0.04$ & $19.6 \pm 0.5$ & $4.21 \pm 0.21$ \\
\hline \hline
\end{tabular}

are summarized in Table 1. Phase shifts for different detector positions were observed and the MIEZE condition was determined by searching the highest contrast. The contrast of the signal in the MIEZE condition was $0.45 \pm 0.04$. This low contrast compared to the beam polarization $(\approx 0.8)$ is considered to be caused by stray fields from the static magnetic field coils of the RSFs. Note that the limitation of the detector thichkness $d$ to observe MIEZE signals is estimated as $d \ll 2 \pi v / \omega_{\mathrm{M}} \simeq 1.3 \mathrm{~mm}$ for $v=66 \mathrm{~m} / \mathrm{s}(\lambda=6 \mathrm{~nm})$ and $\omega_{\mathrm{M}}=2 \pi \times 50 \mathrm{kHz}$, while the scintillator thickness was $0.2 \mathrm{~mm}$. Thus we concluded that the detector thickness was not a major factor of the contrast decreasing.

Figure 6(a) shows the TOF-MIEZE signal with an effective frequency of 

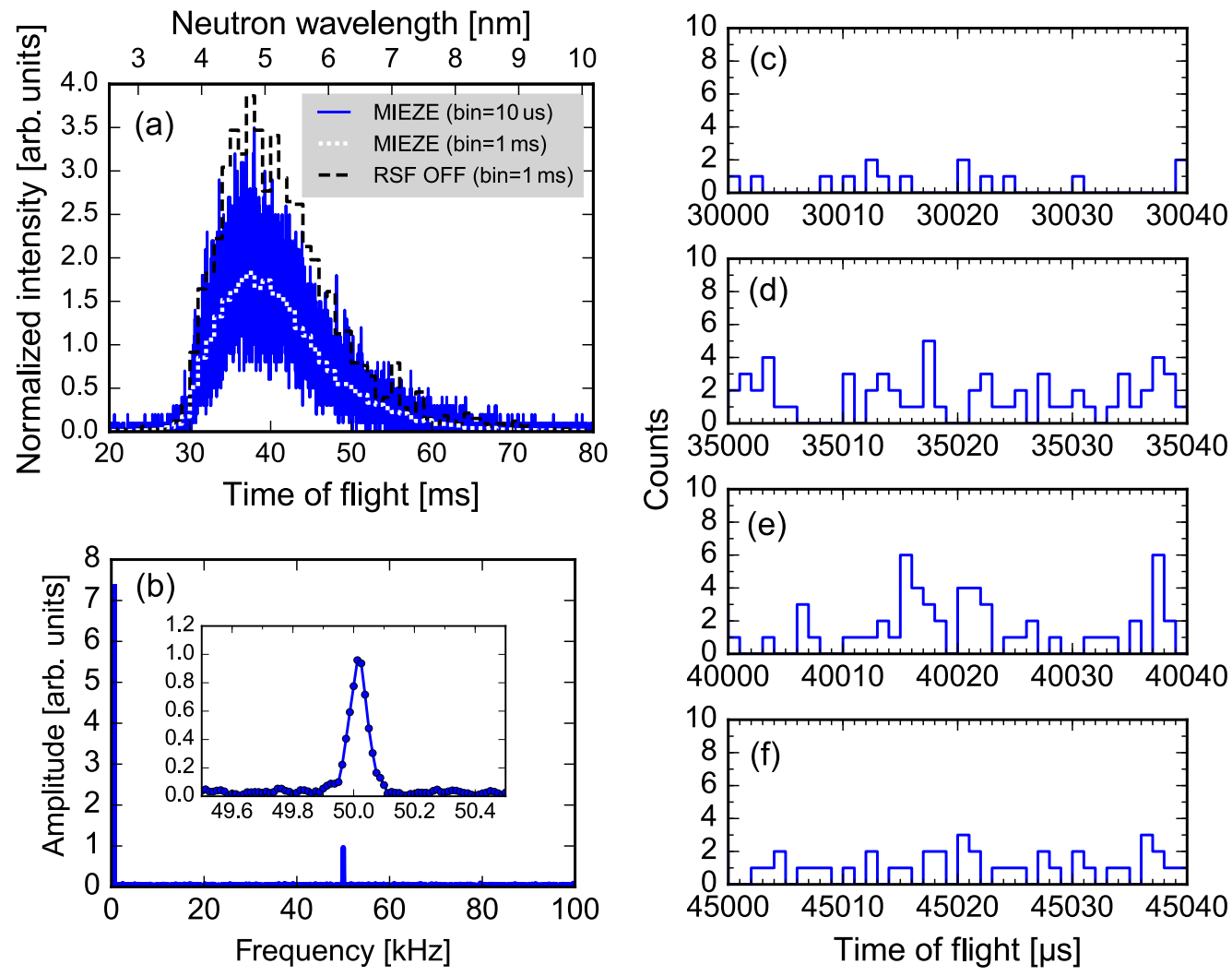

Figure 6: (Left): (a) Spectrum of the direct beam (dashed line), $50 \mathrm{kHz}$ TOF-MIEZE signal with time bin $10 \mu \mathrm{s}$ (solid line), and time-averaged display of the TOF-MIEZE signal with a wide time bin of $1 \mathrm{~ms}$ (dotted line), to be equivalent to $f_{0}(v) / 2$. (b) Fourier transformed amplitude spectrum of the TOF-MIEZE signal. Inset is a magnification of the signal between $49.5-50.5 \mathrm{kHz}$. (Right): Raw data of two periods of the TOF-MIEZE signal in typical TOF ranges: (c) $30-32 \mathrm{~ms}(\lambda=4.1 \mathrm{~nm})$, (d) $35-37 \mathrm{~ms}(\lambda=4.7 \mathrm{~nm})$, (e) 40-42 $\mathrm{ms}(\lambda=5.4 \mathrm{~nm})$, (f) $45-47 \mathrm{~ms}(\lambda=6.1 \mathrm{~nm})$. In these raw data, neutron counts were too low to see any signal. 
$50 \mathrm{kHz}$ (solid line) and a TOF spectrum of the direct beam, i.e., polarized VCN beam without RSF operation (dashed line). The histogram with a wide time bin of $1 \mathrm{~ms}$ for MIEZE (dotted line) shows a time-averaged TOF-MIEZE signal, which corresponds to the half-intensity of the original spectrum, $f_{0}$, in consistency with Eq. (9). The measurement time was $50 \mathrm{~h}$. Figure6(b) is the amplitude spectrum converted from the TOF-MIEZE signal in Figure 6(a) by Fourier transform over the whole TOF frame. A clear peak at $50 \mathrm{kHz}$ in the frequency domain was observed.

Figure 6(c-f) shows the raw neutron counts of the TOF-MIEZE signal with a time bin of $1 \mu \mathrm{s}$ in the TOF ranges. In this experiment, with such a narrow binning, less than 10 neutron counts were recorded in each time bin. To determine the sinusoidal function of an MIEZE signal from the raw data, we summed the neutron counts with the time period of the MIEZE frequency that was determined using Fourier transform (Figure 6(b)). We selected four representative points of TOF: (a) $t_{0}=30 \mathrm{~ms}$, (b) $t_{0}=35 \mathrm{~ms}$, (c) $t_{0}=40 \mathrm{~ms}$, and (c) $t_{0}=45 \mathrm{~ms}$, and accumulated neutron counts over a TOF range of $2 \mathrm{~ms}$ with a repetition period of $40 \mu \mathrm{s}$ (two periods of MIEZE signal) for the four points. Thus the number of accumulation was $50(=2000 / 40)$.

The processed data were the following arrays of neutron counts $I(t)$,

$$
\begin{aligned}
& {\left[I\left(t_{0}\right), I\left(t_{0}+\Delta\right), \cdots, I\left(t_{0}+(n-1) \Delta\right)\right]} \\
& {\left[I\left(t_{0}+T_{\mathrm{M}}\right), I\left(t_{0}+T_{\mathrm{M}}+\Delta\right), \cdots, I\left(t_{0}+T_{\mathrm{M}}+(n-1) \Delta\right)\right]} \\
& \quad \vdots \\
& {\left[I\left(t_{0}+(\nu-1) T_{\mathrm{M}}\right), I\left(t_{0}+(\nu-1) T_{\mathrm{M}}+\Delta\right), \cdots,\right.} \\
& \left.\quad I\left(t_{0}+(\nu-1) T_{\mathrm{M}}+(n-1) \Delta\right)\right] .
\end{aligned}
$$

Here, $\nu=50, n=40, \Delta=1 \mu \mathrm{s}, T_{\mathrm{M}}=40 \mu \mathrm{s}$. Each row, for example the first one: $\left[I\left(t_{0}\right), I\left(t_{0}+\Delta\right), \cdots, I\left(t_{0}+(n-1) \Delta\right)\right]$, corresponds to a histogram shown in Figure 6(c), (d), (e), or (f). These raw data do not show sufficient statistics to see any oscillation. By accumulating counts in each column in the above arrays (Eq. (10)), we obtained the following $n$-length array.

$$
\left[\sum_{k=0}^{\nu-1} I\left(t_{0}+k T_{\mathrm{M}}\right), \sum_{k=0}^{\nu-1} I\left(t_{0}+\Delta+k T_{\mathrm{M}}\right), \cdots, \sum_{k=0}^{\nu-1} I\left(t_{0}+(n-1) \Delta+k T_{\mathrm{M}}\right)\right]
$$

According to this data accumulation, the sinusoidal oscillations of TOFMIEZE signal were reproduced as shown in Fig. 7. The time bin width 


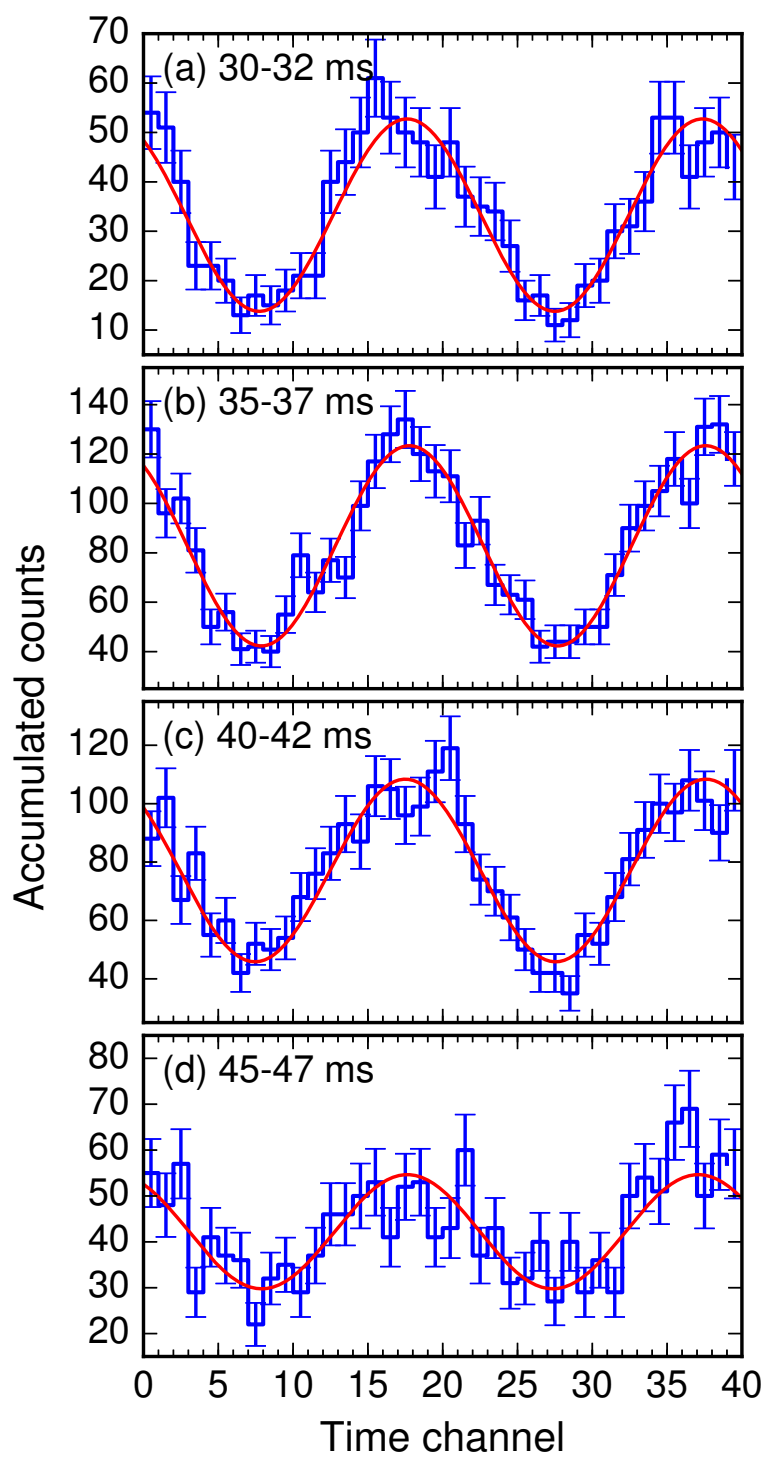

Figure 7: Neutron counts accumulated with a repetition period of $40 \mu$ s over TOF ranges (a) 30-32 $\mathrm{ms}(\lambda=4.1 \mathrm{~nm})$, (b) $35-37 \mathrm{~ms}(\lambda=4.7 \mathrm{~nm})$, (c) $40-42 \mathrm{~ms}(\lambda=5.4 \mathrm{~nm})$, and (d) $45-47 \mathrm{~ms}(\lambda=6.1 \mathrm{~nm})$. The time channel width is $1 \mu \mathrm{s}$. 
Table 2: Fitting parameters and calculated contrasts for each TOF range and corresponding neutron wavelength. The fit function was $y(x)=B+A \sin \left(2 \pi x / T+\phi_{0}\right)$.

\begin{tabular}{cccccc}
\hline \hline & TOF $[\mathrm{ms}]$ & wavelength $[\mathrm{nm}]$ & Contrast, $A / B$ & Period, $T[\mu \mathrm{\mu s}]$ & Phase, $\phi_{0}[\mathrm{rad}]$ \\
\hline (a) & $30-32$ & $4.0-4.2$ & $0.59 \pm 0.04$ & $19.8 \pm 0.4$ & $2.25 \pm 0.12$ \\
(b) & $35-37$ & $4.6-4.9$ & $0.49 \pm 0.03$ & $19.8 \pm 0.3$ & $2.21 \pm 0.09$ \\
(c) & $40-42$ & $5.3-5.5$ & $0.41 \pm 0.03$ & $20.1 \pm 0.4$ & $2.38 \pm 0.13$ \\
(d) & $45-47$ & $5.9-6.2$ & $0.29 \pm 0.03$ & $19.5 \pm 0.5$ & $2.16 \pm 0.21$ \\
\hline \hline
\end{tabular}

of the histograms is 1 us. The signal contrasts and observed parameters at the four TOF ranges are summarized in Table 2. For the different TOF ranges, the phases, $\phi_{0}$ are in agreement within the standard deviation. This implies the validity of the simple data accumulation where we assume that the phase of MIEZE signal is fixed over the whole TOF frame. These contrasts showed a wavelength-dependency. This may have been caused by the stray magnetic fields from the RSF coils, as suggested by the low contrast of the MIEZE signal with the monochromatic beam. In addition, the timemodulation function of the RF amplitude of RSFs given by Eq. (5) was not fully optimized, because the linearity of the RF amplifier system was not perfect.

In this experimental set-up, it was possible to set the distance between the sample and detector to $L_{\mathrm{sd}}=0.5 \mathrm{~m}$. Thus the Fourier times of the TOFMIEZE instrument with $\omega_{\mathrm{M}} / 2 \pi=50 \mathrm{kHz}$ were estimated to be $\tau_{\mathrm{M}}=20 \mathrm{~ns}$ and $70 \mathrm{~ns}$ for VCNs with wavelengths of $\lambda=4 \mathrm{~nm}$ and $6 \mathrm{~nm}$, respectively. Owing to the insufficient performance of the spin flippers for VCNs, we was not able to obtain an effective frequency higher than $50 \mathrm{kHz}$. If we have an effective frequency of $400 \mathrm{kHz}$, which is modest for cold neutrons, and a sample-detector distance of $1.0 \mathrm{~m}$, the Fourier time is calculated to be $1.1 \mu \mathrm{s}$ for $\lambda=6 \mathrm{~nm}$.

\section{Concluding remarks}

The first test experiment of the TOF-MIEZE technique with a VCN beam was performed at the PF2 at ILL towards a high-resolution spectrometer. We observed a TOF-MIEZE signal with an effective frequency of $50 \mathrm{kHz}$ for a wavelength band of 4-6 nm. The maximum Fourier time was estimated to be $70 \mathrm{~ns}$ for the direct beam without a sample. With proper data accumulation, the TOF-MIEZE signals were reproduced from the raw data in which each 
time channel has insufficient event statistics. Fine binning of time channels is required to observe a high-frequency MIEZE signal and the fine binning involves low statistics in each time bin even with an intense pulsed neutron source. The present study indicates the need for this type of data processing in future TOF-MIEZE measurements. The TOF-MIEZE technique can offer an optimized wavelength accumulation range for the required resolution of momentum transfer, $\Delta Q / Q$, because the TOF resolution to define $Q$-value required in most NSE experiments are larger than multiple periods of MIEZE signal with practical frequencies $(\gtrsim 10 \mathrm{kHz})$.

The highest signal contrasts were $0.45 \pm 0.04$ and $0.59 \pm 0.04$ for the MIEZE signal with the monochromatic beam $(\lambda=5 \mathrm{~nm}, \Delta \lambda / \lambda \approx 6 \%)$ and the accumulated TOF-MIEZE signal with the pulsed beam $(\lambda=4.0-4.2 \mathrm{~nm})$, respectively, while the polarization of the $\mathrm{VCN}$ beam was estimated $\approx 0.8$. These contrasts decreasing and wavelength-dependency are considered to be caused by stray fields from the static magnetic filed coil of the RSFs and an incomplete tuning of the time-modulated RF amplitude of the RSFs. We were not able to perform significant measurements on a sample due to an insufficient intensity of neutrons at the sample caused by an absence of focusing optics as well as the relatively high background in this set-up. The use of focusing optics can compensate the VCN intensity effectively and may offer a data correction on MIEZE signals according to the neutron flight path lengths in a focusing set-up. Further investigation of RSF coils dedicated to VCNs and the VCN beam set-up is required with the aim of including beamfocusing devices in future TOF-MIEZE measurements.

\section{Acknowledgment}

The authors are grateful to T. Brenner at PF2 of ILL for his technical support. This work was supported by Japan Society for the Promotion of Science (JSPS) KAKENHI Grant Nos. JP23360428 and JP15K13405. Certain device developments were supported by the program of Development of System and Technology for Advanced Measurement Analysis (SENTAN), JST, and the Neutron Scattering Program Advisory Committee of IMSS, KEK (Proposal Nos. 2009S07 and 2014S07). T.O. acknowledges the financial support by Grant-in-Aid for JSPS Fellows. H.F. is supported by the Austrian Science Fund FWF under the Doctoral Program W1252-N27 Particles and Interactions and under grant P26781-N20. 


\section{References}

[1] J. Byrne, Neutrons, nuclei, and matter: an exploration of the physics of slow neutrons, Vol. : hbk, Institute of Physics Pub., Bristol, 1994.

[2] M. Hino, S. Tasaki, Y. Kawabata, T. Ebisawa, P. Geltenbort, T. Brenner, J. S. Butterworth, R. Gähler, N. Achiwa, M. Utsuro, Development of a very cold neutron spin interferometer at the ill, Physica B 335 (14) (2003) 230-233. doi:10.1016/S0921-4526(03)00244-8.

[3] J. Klepp, C. Pruner, Y. Tomita, K. Mitsube, P. Geltenbort, M. Fally, Mirrors for slow neutrons from holographic nanoparticle-polymer freestanding film-gratings, Appl. Phys. Lett. 100 (21) (2012) 214104. doi: $10.1063 / 1.4720511$.

[4] H. Abele, The neutron. its properties and basic interactions, Prog. Part. Nucl. Phys. 60 (1) (2008) 1-81. doi:10.1016/j.ppnp.2007.05.002.

[5] G. van der Zouw, M. Weber, J. Felber, R. Ghler, P. Geltenbort, A. Zeilinger, Aharonovbohm and gravity experiments with the verycold-neutron interferometer, Nuclear Instruments and Methods in Physics Research Section A: Accelerators, Spectrometers, Detectors and Associated Equipment 440 (3) (2000) 568-574. doi:10.1016/ S0168-9002(99)01038-4.

[6] A. I. Erko, I. Mourad, K. Thomas, M. Alan G, Modern developments in X-ray and neutron optics, Springer series in optical sciences 137, Springer, Berlin, 208.

[7] G. E. Ice, C. R. Hubbard, B. C. Larson, J. W. L. Pang, J. D. Budal, S. Spooner, S. C. Vogel, Kirkpatrick-baez microfocusing optics for thermal neutrons, Nucl. Instrm. Meth. A 539 (1-2) (2005) 312-320. doi:10.1016/j.nima.2004.10.005.

[8] K. Yamamura, M. Nagano, H. Takai, N. Zettsu, D. Yamazaki, R. Maruyama, K. Soyama, S. Shimada, Figuring of plano-elliptical neutron focusing mirror by local wet etching, Opt. Express 17 (8) (2009) 6414-6420. doi:10.1364/0E.17.006414.

[9] D. Yamazaki, M. Nagano, R. Maruyama, H. Hayashida, K. Soyama, K. Yamamura, Neutron focusing by a kirkpatrickbaez type super-mirror, JPS Conf. Proc. 8 (2015) 051009. doi:10.7566/JPSCP.8.051009. 
[10] D. Liu, B. Khaykovich, M. V. Gubarev, J. Lee R., L. Crow, B. D. Ramsey, D. E. Moncton, Demonstration of a novel focusing small-angle neutron scattering instrument equipped with axisymmetric mirrors, Nat. Commun. 4. doi:10.1038/ncomms3556.

[11] J. Guo, S. Takeda, S. Morita, M. Hino, T. Oda, J. Kato, Y. Yamagata, M. Furusaka, New fabrication method for an ellipsoidal neutron focusing mirror with a metal substrate, Opt. Express 22 (20) (2014) 24666-24677. doi:10.1364/0E.22.024666.

[12] S. Takeda, Y. Yamagata, N. L. Yamada, M. Hino, T. Hosobata, J. Guo, S. Morita, T. Oda, M. Furusaka, Development of a large plano-elliptical neutron-focusing supermirror with metallic substrates, Opt. Express 24 (12) (2016) 12478-12488. doi:10.1364/0E.24.012478.

[13] M. Yamada, Y. Iwashita, M. Ichikawa, Y. Fuwa, H. Tongu, H. M. Shimizu, K. Mishima, N. L. Yamada, K. Hirota, Y. Otake, Y. Seki, Y. Yamagata, M. Hino, M. Kitaguchi, U. Garbe, S. J. Kennedy, W. Tung Lee, K. H. Andersen, B. Guerard, G. Manzin, P. Geltenbort, Pulsed neutron-beam focusing by modulating a permanent-magnet sextupole lens, Prog. Theor. Exp. Phys. 2015 (4). doi:10.1093/ptep/ ptv015.

[14] F. Mezei, Neutron spin echo: A new concept in polarized thermal neutron techniques, Z. Phys. 255 (1972) 146-160. doi:10.1007/ BF01394523.

[15] F. Mezei (Ed.), Neutron Spin Echo, Vol. 128 of Lecture Notes in Physics, Springer-Verlag, Berlin Heidelberg, 1980.

[16] F. Mezei, C. Pappas, T. Gutberlet (Eds.), Neutron Spin Echo Spectroscopy: Basics, Trends and Applications, Vol. 601 of Lecture Notes in Physics, Springer, Berlin Heidelberg, 2003.

[17] R. Gähler, R. Golub, T. Keller, Neutron resonance spin-echo - a new tool for high-resolution spectroscopy, Physica B 180 (1992) 899-902. doi : 10.1016/0921-4526(92) 90503-K.

[18] R. Georgii, G. Brandl, N. Arend, W. Häussler, A. Tischendorf, C. Pfleiderer, P. Böni, J. Lal, Turn-key module for neutron scattering with submicro-ev resolution, Appl. Phys. Lett. 98 (7). doi:10.1063/1.3556558. 
[19] J. Kindervater, N. Martin, W. Häußler, M. Krautloher, C. Fuchs, S. Muehlbauer, J. A. Lim, E. Blackburn, P. Böni, C. Pfleiderer, Neutron spin echo spectroscopy under $17 \mathrm{t}$ magnetic field at reseda, EPJ Web of Conferences 83. doi:0300810.1051/epjconf/20158303008.

[20] H. Hayashida, M. Hino, M. Kitaguchi, N. Achiwa, Y. Kawabata, A new mieze technique for investigating relaxation of magnetic nanoparticles, Nucl. Instrm. Meth. A 600 (1) (2009) 56-59. doi:10.1016/j.nima. 2008.11.101.

[21] P. Hank, W. Besenböck, R. Gähler, M. Köppe, Zero-field neutron spin echo techniques for incoherent scattering, Physica B 234 (1997) 11301132. doi:10.1016/S0921-4526(97)89269-1.

[22] W. Häußler, L. Kredler, Dynamic neutron scattering on incoherent systems using efficient resonance spin flip techniques, AIP Conference Proceedings 1599 (2014) 298-301. doi:10.1063/1.4876837.

[23] H. Hayashida, M. Hino, M. Kitaguchi, Y. Kawabata, N. Achiwa, A study of resolution function on a mieze spectrometer, Meas. Sci. Technol. 19 (3). doi:10.1088/0957-0233/19/3/034006.

[24] G. Brandl, R. Georgii, W. Häußler, S. Muehlbauer, P. Böni, Large scaleslong times: Adding high energy resolution to sans, Nucl. Instrum. Meth. A 654 (1) (2011) 394-398. doi:10.1016/j.nima.2011.07.003.

[25] T. Weber, G. Brandl, R. Georgii, W. Häußler, S. Weichselbaumer, P. Böni, Monte-carlo simulations for the optimisation of a tof-mieze instrument, Nucl. Instrm. Meth. A 713 (2013) 71-75. doi:10.1016/j. nima.2013.03.010.

[26] M. Hino, M. Kitaguchi, H. Hayashida, Y. Kawabata, S. Tasaki, T. Ebisawa, D. Yamazaki, R. Maruyama, K. Tanaka, N. Torikai, R. Inoue, T. Kanaya, A test of mieze-reflectometer for study of surface and interface, Physica B 385386, Part 2 (2006) 1125-1127. doi:10.1016/j. physb.2006.05.388.

[27] W. Besenböck, R. Gähler, P. Hank, R. Kahn, M. Köppe, C. H. de Novion, W. Petry, J. Wuttke, First scattering experiment on mieze: a fourier transform time-of-flight spectrometer using resonance coils, 
Journal of Neutron Research 7 (1) (1998) 65-74. doi:10.1080/ 10238169808200231 .

[28] W. Häußler, O. Holderer, T. Unruh, J. Wuttke, High-resolution neutron spectroscopy at the frm ii, Neutron News 22 (3) (2011) 24-30. doi: $10.1080 / 10448632.2011 .598804$.

[29] R. Georgii, P. Böni, M. Janoschek, C. Schanzer, S. Valloppilly, Mira - a flexible instrument for vcn, Physica B (2007) 150-152doi:DOI10.1016/ j.physb.2007.02.088.

[30] T. Oda, M. Hino, M. Kitaguchi, P. Geltenbort, Y. Kawabata, Pulsed neutron time-dependent intensity modulation for quasi-elastic neutron scattering spectroscopy, Rev. Sci. Instrum. 87 (10) (2016) 105124. doi: $10.1063 / 1.4965835$.

[31] M. Bleuel, M. Broll, E. Lang, K. Littrell, R. Gähler, J. Lal, First tests of a mieze (modulated intensity by zero effort)-type instrument on a pulsed neutron source, Physica B 371 (2) (2006) 297-301. doi:10. 1016/j.physb.2005.10.124.

[32] G. Brandl, J. Lal, J. Carpenter, L. Crow, L. Robertson, R. Georgii, P. Böni, M. Bleuel, Tests of modulated intensity small angle scattering in time of flight mode, Nucl. Instrum. Meth. A 667 (2012) 1-4. doi: 10.1016/j.nima.2011.11.075.

[33] G. Cicognani (Ed.), The ILL Yellow Book, Institut Laue-Langevin, Grenoble, 2008.

URL https://www.ill.eu/en/instruments-support/ instruments-groups/yellowbook/

[34] M. Hino, T. Oda, M. Kitaguchi, N. L. Yamada, S. Tasaki, Y. Kawabata, The ion beam sputtering facility at KURRI: Coatings for advanced neutron optical devices, Nucl. Instrm. Meth. A 797 (2015) 265-270. doi:10.1016/j.nima.2015.06.046.

[35] K. Hirota, T. Shinohara, K. Ikeda, K. Mishima, T. Adachi, T. Morishima, S. Satoh, T. Oku, S. Yamada, H. Sasao, J. Suzuki, H. M. Shimizu, Development of a neutron detector based on a positionsensitive photomultiplier, Phys. Chem. Chem. Phys. 7 (2005) 1836-1838. doi:10.1039/B417838F, 
[36] N. Ramsey, Molecular beams, The International series of monographs on physics, Clarendon Press, Oxford, 1956.

[37] R. Maruyama, T. Ebisawa, S. Tasaki, M. Hino, M. Takeda, T. Kawai, Y. Kawabata, K. Sakai, A resonance neutron-spin flipper for neutron spin echo at pulsed sources, Physica B 335 (2003) 238-242. doi:10. 1016/S0921-4526(03) 00246-1.

[38] H. Hayashida, M. Kitaguchi, M. Hino, Y. Kawabata, R. Maruyama, T. Ebisawa, Development of a resonance spin flipper for nrse/mieze on a pulsed neutron beam with an oscillating frequency of $500 \mathrm{khz}$, Nucl. Instrm. Meth. A 574 (2) (2007) 292-296. doi:10.1016/j.nima. 2007. 01.179 .

[39] R. Golub, R. Gähler, T. Keller, A plane-wave approach to particlebeam magnetic-resonance, Am. J. Phys. 62 (9) (1994) 779-788. doi: 10.1119/1.17459. 